\title{
Delineamento e Avaliação de um Programa de Adaptação Acadêmica no Ensino Superior
}

\author{
Marcela de Moura Franco Barbosa ${ }^{1}$ \\ Marina Cardoso de Oliveira \\ Universidade Federal do Triângulo Mineiro, Uberaba-MG, Brasil \\ Lucy Leal Melo-Silva \\ Universidade de São Paulo, Ribeirão Preto-SP, Brasil \\ Maria do Céu Taveira \\ Universidade do Minho, Braga, Portugal
}

\section{Resumo}

A adaptação acadêmica é um processo complexo e seu êxito relaciona-se com a satisfação com a vida e sucesso na carreira. Este estudo buscou delinear e apresentar os resultados de um programa de adaptação acadêmica no ensino superior. Participaram 22 universitários com dificuldades de adaptação. Para o delineamento recorreu-se ao método da pesquisa-ação e para a avaliação dos resultados inspirou-se no método quase-experimental com a avaliação antes e após a intervenção. Os resultados mostraram que com o delineamento foi possível disponibilizar um modelo de oficina que atende às demandas do público-alvo. Em relação aos resultados da intervenção constatou-se que os participantes conseguiram melhorar seus escores após a intervenção em importantes dimensões da Escala de Ajustamento Acadêmico e de Adaptabilidade de Carreira.

Palavras-chave: Adaptação, universitários, orientação profissional e de carreira

\section{Abstract: Design and Evaluation of a Higher Education Academic Adaptation Program}

Academic adaptation is a complex process and its success is related to life satisfaction and career success. This study aimed to design and evaluate the results of an Academic Adaptation Program in higher education. Twenty-two university students, with problems of adaptation, participated in the study. For the design stage, the action-research method was applied. For the assessment, we were inspired by the quasi-experimental method with the comparison before and after the intervention. As a result, in the design stage was offered a workshop model that met the demands of the target group. Regarding the assessment of the intervention, participants were able to improve their scores after the intervention in important dimensions of the Academic Adjustment Scale and Career Adaptability.

Keywords: Adaptation, undergraduate students, professional and career guidance

\section{Resumen: Diseño y evaluación de un Programa de Adaptación Académica en la enseñanza superior}

La adaptación académica es un proceso complejo y su éxito se relaciona con la satisfacción con la vida y el éxito en la carrera. Este estudio buscò delinear y evaluar los resultados de un Programa de Adaptación Académica en la enseñanza superior. Participaron 22 universitarios con problemas de adaptación. Para cumplir los objetivos, se recurrió al método de investigación-acción y al diseño cuasi-experimental con evaluación antes y después de la intervención. Los resultados mostraron que con el programa fue posible disponibilizar un modelo de intervención grupal en correspondencia con las demandas del grupo meta. En relación con los resultados de la intervención, se constató que los participantes lograron mejorar sus puntuaciones después de la intervención em importantes dimensiones de la Escala de Adaptación Académica y de Adaptabilidad de Carrera.

Palabras clave: Adaptación, universitarios, orientación profesional y de carrera

\footnotetext{
${ }^{1}$ Endereço para correspondência: Avenida Getúlio Guaritá, 159, Abadia, 38025-440, Uberaba, MG. E-mail: mco.uftm@gmail.com
} 
Nos últimos vinte anos foi possível observar aumento no número de pessoas que ingressaram e concluíram o ensino superior em todo o mundo (OCDE, 2015). No Brasil, no período entre 2005 e 2015 o número de matrículas na educação superior aumentou $75,7 \%$. Nos últimos 10 anos, as matrículas praticamente dobraram, passando de 4,6 milhões para mais de oito milhões de alunos. Concomitantemente à expansão da oferta de vagas, os dados apontam para o crescimento da evasão no ensino superior. Em 2010, 11,4\% dos alunos abandonaram o curso no qual ingressaram e, em 2014, esse número chegou a 49\% [Instituto Nacional de Estudos e Pesquisas Educacionais Anísio Teixeira (INEP), 2016].

Em resposta a esta fase de expansão e de maior acesso ao ensino superior, impõe-se o desafio de assegurar a permanência dos alunos, o seu desenvolvimento psicossocial, a qualidade da sua formação e a conclusão efetiva da graduação (Almeida, Araújo, \& Martins, 2016), uma vez que o país precisa garantir acesso e permanência da população em todos os níveis educacionais. Para tal, faz-se necessário uma atuação conjunta de órgãos governamentais e das instituições de ensino para melhorar a adaptação desses jovens à educação superior. Porém, ainda são escassas e pouco sistematizadas as iniciativas nessa direção (Fagundes, Luce, \& Espinar, 2014; Oliveira, Detomini, \& Melo-Silva, 2013).

Ingressar na universidade representa um marco na vida pessoal, pois estar no ensino superior é uma oportunidade de concretização de projetos de vida pessoal, profissional e familiar. Essa oportunidade configura-se com muitos desafios, explicitando diferentes sentimentos, como medo, ansiedade e insegurança e, isto exigirá habilidades de adaptação dos estudantes (Soares \& Del Prette, 2015). Trata-se, portanto, da transição do ensino médio para o superior, da adolescência para a adultez, com todos os desafios que esta etapa do ciclo vital requer em termos biológicos, psicológicos e sociais. Além disso, há expectativas sociais e exigências acadêmicas para a realização de um curso de nível superior de ensino, muitas vezes sentidas pelos universitários como pressão. Assim, ao vivenciarem a universidade com projetos mal definidos os universitários podem sofrer inúmeros problemas de adaptação que se manifestam de diferentes formas, tais como insatisfação, baixo rendimento acadêmico, problemas de saúde e evasão (Byrd \&McKinney, 2012; Freitas \& Oliveira, 2017; Oliveira \& Morais, 2015). E, em respostas desadaptadas frente às demandas do contexto universitário (Oliveira, Santos, \& Dias, 2016). Por outro lado, o contexto acadêmico exige que os alunos se adaptem às novas formas de aprendizagem por meio das iniciativas pessoais de autorregulação (Almeida, 2007).
As pesquisas sobre a transição do ensino médio para o ensino superior, na visão de Fagundes et al. (2014) têm priorizado três dimensões do fenômeno. A primeira é a dimensão pessoal que inclui as características dos estudantes, os perfis pessoais, a trajetória educativa, a capacidade de enfrentamento a diferentes situações de relacionamentos interpessoais, as expectativas sociais, o apoio familiar, as reações físicas, psicossomáticas e o estado de humor. A segunda dimensão, a institucional refere-se prioritariamente às questões relacionadas ao sistema educativo, às condições e características da instituição, como a oferta curricular, qualidade do ensino, infraestrutura, professores, entre outras condições. E a terceira dimensão, a contextual, que se relaciona com as políticas educativas e socioeconômicas, que direta ou indiretamente afetam a vida universitária.

Considerando as dimensões supracitadas, a adaptação pode ser entendida como o resultado de processos cognitivos, afetivos, institucionais e sociais que quando harmonizados, tornam mais provável o êxito do aluno, o aproveitamento efetivo das condições que a universidade oferece, um melhor desenvolvimento pessoal e ajustamento ao contexto acadêmico e social (e.g., Fagundes et al., 2014; Guerreiro-Casanova \& Polydoro, 2011; Soares \& Del Prete, 2015). Diante do exposto, fica evidente a necessidade de as instituições de ensino superior oferecerem ajuda e suporte aos estudantes no processo de adaptação ao contexto universitário, no sentido de facilitar a obtenção de bons resultados em suas trajetórias acadêmicas, pessoais e profissionais (Fagundes et al., 2014; Freitas \& Oliveira, 2017).

Neste sentido, existe a demanda pelo delineamento e a avaliação de programas de orientação acadêmica e de carreira direcionados aos estudantes universitários. Apesar da sua relevância psicossocial, são poucas as iniciativas nessa direção (Teixeira \& Gomes, 2004). Em um cenário de velozes e vertiginosas mudanças: (a) no mundo do trabalho, incluindo a flexibilização na legislação; (b) nas relações interpessoais, sobretudo mediadas pelas redes sociais; e (c) no aumento da incidência de doenças mentais; entre outras [Berhein \& Chauí, 2008; World Health Organization (WHO), 2017]; tornou-se premente a assistência psicológica à população em geral e, em particular, aos estudantes universitários (Byrd \&McKinney, 2012), foco deste estudo.

De modo geral, as demandas dos alunos se configuram em quatro vertentes, organizadas para fins didáticos, pois algumas delas podem se relacionar. As vertentes requerem distintas estratégias ou intervenções: (a) acadêmica, relacionados a questões de adaptação à vida universitária, dificuldades na autorregulação da aprendizagem, relação professor-aluno, evasão, (des)engajamento com o curso, entre outras; (b) de carreira, relacionadas à escolha e construção da 
vida/carreira, envolvimento com atividades acadêmicas, de extensão, de iniciação científica, intercâmbio, estágios, mudança de curso, transição para o mercado ou a pós-graduação, trabalho do futuro ou futuro do trabalho; (c) de saúde física, psíquica ou emocional, como por exemplo relacionamentos interpessoais, afastamentos por motivo de saúde, envolvimento com drogas, depressão, necessidade de atendimento psicológico e psiquiátrico, para onde encaminhar, quando acionar os familiares, entre outras questões de promoção de saúde ou de tratamento; e (d) questões específicas, relativas à discriminação, assédio e violência contra mulheres e demais questões de gênero e de exclusão.

Este estudo se insere na vertente acadêmica, e portanto do delineamento e da avaliação de um Programa de Adaptação Acadêmica no Ensino Superior. Para que programas desta natureza sejam desenvolvidos e bem delineados, correspondendo às exigências deste século, bem como ao enfrentamento de problemas de adaptação acadêmica, Dois referenciais teóricos foram considerados apropriados para subsidiar este estudo, a Teoria Sociocognitiva de Ajustamento Acadêmico (Lent, 2004) e o modelo de intervenção do Paradigma Life Design (Nota, Soresi, Ferrari, \& Ginevra, 2014).

Nos aspectos relacionados à adaptação acadêmica, a Teoria Sociocognitiva de Ajustamento Acadêmico proposta por Lent (2004) é relevante, pois esse modelo baseia-se na premissa de que um conjunto de recursos pessoais e sociais permitem às pessoas desenvolverem os atributos que desejam para melhorarem suas vidas e transformarem seus sistemas sociais. De acordo com o modelo, o processo de adaptação ao ambiente do ensino superior deve aliar conceitos como: bem-estar, ajustamento à vida acadêmica e satisfação com a vida em geral, levando também em consideração variáveis pessoais, como a personalidade e a autoeficácia que o estudante tem para superar os desafios acadêmicos e aprender novos conceitos. Por sua vez, o modelo do Paradigma Life Design pressupõe o aconselhamento de carreira como uma estratégia que possibilita à pessoa que ela compreenda a construção de sua carreira, a partir de um conjunto de recursos psicossociais que são úteis para lidar com as exigências do mundo do trabalho na vida pós-moderna (Nota et al., 2014). Por meio deste referencial, é possível orientar os estudantes na graduação, ou até antes dela, a começarem a rascunhar as trajetórias de vida que querem construir. $\mathrm{Na}$ intervenção analisada neste estudo, a premissa é de que os universitários possam compreender a construção gradual de sua vida/carreira (passado, presente e futuro), incluindo a tomada de consciência e o engajamento em projetos da própria universidade que facilitam imaginar projetos para a vida acadêmica e após a graduação.
Dessa forma, as intervenções que buscam facilitar a adaptação acadêmica devem se concentrar no desenvolvimento e na consolidação de recursos psicossociais que ajudem os estudantes a criarem estratégias de enfrentamento para as situações que se apresentam como desafios, em vez de apenas fornecerem informações. Devem ser capazes de ajudar as pessoas a criarem recursos para se tornarem seus próprios especialistas no processo de entendimento da situação e de resolução de problemas para a conquista de metas futuras. Uma das atribuições do mediador é a de encorajar os universitários para se imaginarem em diferentes papéis de vida, a lidarem com barreiras na execução dos projetos de vida e a construírem redes de apoio social, como apontam Nota et al. (2014).

Diante do exposto, pode-se afirmar que a adaptação dos jovens no ensino superior é um processo progressivo e para que ocorra são exigidos dos estudantes conhecimentos e habilidades em aspectos da autorregulação da aprendizagem, autoeficácia e adaptabilidade de carreira, sejam nos estudos ou na forma de lidar e enfrentar problemas (Almeida, 2007; Nota et al, 2014; Soares \& Del Prette, 2015). Tais habilidades podem ser ativadas por meio dos programas de adaptação acadêmica, fazendo-se necessário a criação desses serviços nas universidades. Além da criação e implementação desses programas, faz-se necessário desenvolver estratégias de avaliação das intervenções com o objetivo de verificar o processo e os resultados, com vistas à manutenção ou ao aprimoramento.

\section{Avaliação de intervenção no contexto de Orientação Profissional e de Carreira}

No contexto dos serviços de Orientação Profissional e de Carreira, a avaliação da intervenção é fundamental para a continuidade dos serviços, como apontado por Melo-Silva e Jacquemin (2001), destacando a necessidade de avalição em três dimensões: inputs, processos e resultados; em um período em que pouco se abordava a avaliação da intervenção no Brasil, tendo sido este estudo pioneiro. Avaliar é preciso, e tal tarefa no contexto brasileiro adquire uma dimensão complexa e desafiadora, como apontado por Almeida e Melo-Silva (2006), Arruda e Melo-Silva (2010), Melo-Silva (2011), Alonso e Melo-Silva (2013).

A avaliação da intervenção pode ser caracterizada como "uma forma de analisar práticas e instrumentos, com o objetivo de tornar o processo de intervenção mais eficaz e adequado a cada cenário e contexto" (Arruda \& Melo-Silva, 2010, p. 225). A avaliação da intervenção desempenha um papel relevante, pois por meio dela obtém-se benefícios como o levantamento de necessidade de aprimoramento do processo e resultados obtidos com a 
intervenção; a garantia da qualidade e otimização dos serviços disponibilizados à sociedade; o auxílio dos participantes por meio de seu envolvimento ativo e reflexivo em todas as etapas do processo de avaliação; a estimulação à mudança, favorecendo a tomada de decisão esclarecida acerca das intervenções e, o maior nível de confiança da população em relação aos serviços prestados (Bardagi \& Albanaes, 2015).

No que diz respeito às avaliações de intervenção no domínio da carreira, Alonso e Melo-Silva (2013) apontam que o mais importante ao criar um modelo de avaliação é levar em consideração as especificidades culturais e sociais do país em que o programa é desenvolvido, assim como, os objetivos propostos e os métodos empregados. Por sua vez, Bardagi e Albanaes (2015) recomendam que para uma efetiva avaliação, devem ser coletadas informações envolvendo as características e os resultados da intervenção, englobando as tomadas de decisões relacionadas à manutenção, melhoria ou eliminação de alguma atividade praticada nos programas. As autoras ressaltam ainda que avaliação de intervenção pode ser realizada em dois âmbitos: avaliação de resultados e de processos. A avaliação dos resultados centra-se nos clientes, indagando até que ponto a mudança pode ser atribuída a uma dada intervenção. Por sua vez, a avaliação do processo busca estabelecer possíveis adaptações no curso da intervenção para melhoria do processo (Bardagi \& Albanaes, 2015), incidindo sobre os mecanismos que ocorrem no interior da intervenção associados aos resultados (Alonso \& Melo-Silva, 2013).

Embora no Brasil esteja crescendo o interesse pelos estudos de avaliação de intervenções no domínio da carreira, ainda se faz necessária a consolidação de uma cultura de avaliação que possa resultar em elevação da eficiência e da eficácia das intervenções. No contexto das transições que envolvem o ensino superior, observa-se a relevância de estudos que busquem sistematizar a implementação e a avaliação de programas que promovam a adaptação acadêmica, foco deste estudo. Espera-se com esta investigação contribuir com a produção do conhecimento no âmbito da Psicologia Vocacional notadamente no domínio das intervenções e respectivas avaliações no cenário universitário. Assim, este estudo teve como objetivo delinear e apresentar os resultados de um programa de adaptação acadêmica no ensino superior.

\section{Método}

\section{Tipo de estudo}

Este estudo caracteriza-se como uma pesquisa-ação com delineamento quase-experimental, com dois momentos de avaliação, antes da intervenção (pré-teste) e após a intervenção (pós-teste), para verificação de possíveis mudanças nos escores dos participantes (Bogdan \& Biklen, 1994; Cozby, 2009).

\section{Participantes}

A amostra foi composta por 22 universitários, que participaram das oficinas desenvolvidas no período em análise, sendo a maioria do sexo feminino $(N=18)$. A faixa etária dos participantes variou de 18 a 25 anos $(M=20,66, D P=1,37)$. A maioria dos participantes estava matriculada no curso de Psicologia $(N=7)$, seguido da Engenharia Ambiental e Engenharia de Produção, ambos com três participantes, Enfermagem com dois, e os demais cursos: Fisioterapia, Engenharia de Alimentos, Medicina, Engenharia Elétrica, Física e Nutrição com um participante cada. O período cursado pela amostra variou do $2^{\circ}$ ao $8^{\circ}$, sendo a maioria $(N=6)$, do $6^{\circ}$ período, seguido por estudantes do $4^{\circ}$ Período $(N=5)$. Entre os participantes, doze (54\%) informaram que o curso no qual se encontravam matriculados não foi a primeira opção de escolha. Em relação à satisfação com o curso, treze participantes (59\%) responderam que estão satisfeitos com a escolha.

\section{Instrumentos}

Formulário de avaliação pessoal de encontro. Criado pelas pesquisadoras para fins da avaliação do programa, foi aplicado ao final de cada encontro, como oportunidade para os participantes responderem dando um feedback do dia. O formulário foi organizado em espaços para os alunos registrarem pontos positivos, dúvidas e sugestões para aperfeiçoamento da intervenção.

Questionário sociodemográfico. Foi construído pela equipe de pesquisadores e teve como objetivo coletar informações gerais sobre as características pessoais, socioeconômicas e acadêmicas dos participantes.

Diários de campo. Estratégia de registro decorrente de observação da intervenção que foram utilizados para o entendimento do processo de intervenção e para fornecer insumos para a reflexão sobre os resultados derivados da aplicação dos instrumentos de medida.

Questionário de Ajustamento Acadêmico - versão adaptada para o Brasil [Lent, et.al. (2005), versão adaptada para o português de Lent, Taveira, Sheu \& Singley (2009)]. Este instrumento avalia a disposição afetiva, a autoeficácia acadêmica, a percepção de alcance e progresso em objetivos acadêmicos, de recursos ou barreiras, a satisfação com a vida acadêmica, o estresse percebido com a vida acadêmica, a percepção de adaptação geral à 
universidade e a satisfação com a vida em geral. Trata-se de um questionário com 56 itens, que se encontram organizados em nove subescalas, sendo sete relacionadas às Experiências Acadêmicas e duas relacionadas à Vida Geral. Na Parte I, sobre as Experiências Acadêmicas, encontra-se a subescala de Autoeficácia acadêmica (5 itens) que avalia o grau de confiança do aluno na sua capacidade de manter-se envolvido no seu curso de graduação ao longo dos próximos dois semestres. A Parte II é composta pela subescala Autoeficácia para lidar com barreiras (7 itens) que avalia a confiança do aluno em lidar com barreiras que pode confrontar ao prosseguir no curso. A Parte III contém a subescala Percepção de alcance e progresso de objetivos acadêmicos (8 itens), que avalia o quanto o aluno tem de progresso até o presente momento no semestre em relação ao envolvimento com o curso, trabalhos, exames e disciplinas. A Parte IV contém a subescala Percepção de apoio social (9 itens) que avalia os tipos de situações que podem sustentar ou apoiar o processo do aluno no curso. Na Parte V a subescala de Satisfação com a vida acadêmica (7 itens), avalia o quão satisfeito o aluno se encontra com a vida acadêmica, curso, aulas e estudos. Na Parte VI a subescala de Percepção de adaptação geral à universidade (1 item) avalia até que ponto o aluno está ajustado com às exigências da universidade. A Parte VII a subescala de Estresse percebido com a vida acadêmica (4 itens) indica os sentimentos e pensamentos ocorridos no último mês em relação a vida acadêmica do aluno. A Parte I da seção Vida Geral, refere-se à Disposição afetiva (10 itens) que descreve um conjunto de palavras relacionadas a diferentes sentimentos e emoções, e o aluno indica em que medida ele se sente em relação às palavras e, Parte II Satisfação geral com a vida (5 itens), onde o aluno avalia sua satisfação geral com vida em relação ao que considera ideal, no alcance da satisfação e dos objetivos de vida. Todos os itens são respondidos numa escala de resposta do tipo Likert. Nas Partes I e II, das Experiências Acadêmicas as opções de resposta variam entre 0 e 9 e, nas Partes III a VII e na Parte I da seção Vida em Geral, as respostas variam entre 1 e 5 e, na Parte II variam entre 1 e 7 . Nas escalas, os valores 0 e 1 significam "Muito pouco", "Nada", "Nunca", "Nenhum progresso", "Nenhuma confiança" ou "Discordo fortemente" e; os valores 5, 7 e/ou 9 significam "Plena confiança", "Concordo fortemente", "Excelente Progresso", "Frequentemente" ou "Extremamente". A tarefa dos participantes consiste em selecionar, na escala de resposta, a opção que melhor traduz o seu grau de ajustamento, adaptação e bem-estar ao seu atual contexto acadêmico. O Questionário apresenta boas qualidades psicrométricas na sua versão adaptada para o Brasil, conforme estudo de Lent, Taveira, Cristiane, Sheu e Pinto (2018).

Escala de Adaptabilidade de Carreira (EAC, originalmente intitulado Career Adapt-Abilities Scale - CAAS), versão adaptada para o Brasil (Audibert \& Teixeira, 2015). Este instrumento avalia os recursos psicossociais relacionados à adaptabilidade de carreira. Trata-se de um questionário constituído por um total de 24 itens, que se encontram organizados em quatro subescalas: (a) Preocupação com a Carreira (6 itens); (b) Controle (6 itens); (c) Curiosidade/Exploração de Carreira (6 itens); (d) Confiança (6 itens). Todos os itens são respondidos numa escala de resposta de tipo Likert e gráfico-numérico, entre 1 e 5 pontos, em que 1 significa "Desenvolvi pouco ou nada", 2 significa "Desenvolvi mais ou menos", 3 significa "Desenvolvi bem", 4 significa "Desenvolvi muito bem" e por último 5, significa "Desenvolvi extremamente bem". A tarefa dos participantes consiste em selecionar, da escala de resposta, a opção que melhor traduz o quanto desenvolveu cada uma das habilidades de acordo com o momento atual do mesmo. A escala tem obtido qualidades psicrométricas satisfatórias para o contexto brasileiro.

\section{Procedimentos de coleta e análise de dados}

Para realização deste estudo, o mesmo foi submetido ao Comitê de Ética em Pesquisa da Universidade sede das duas primeiras autoras e foi aprovado pelo protocolo $\mathrm{n}^{\mathrm{o}} 1.630 .238$, em 2016. Para a coleta de dados, os acadêmicos da Universidade foram informados e convidados a participar voluntariamente do Programa de Adaptação Acadêmica por meio de envio de e-mails institucionais e divulgação do programa nos campi da universidade, bem como pelas redes sociais facebook e instagram. Os estudantes que decidiram participar tiveram que assinar o Termo de Consentimento Livre e Esclarecido (TCLE).

Em relação ao delineamento do programa, inicialmente, com base nos pressupostos teóricos adotados - Teoria Sociocognitiva de Ajustamento Acadêmico e Paradigma Life Design -, foi estruturada uma intervenção, no formato de oficina, conforme descrito na Tabela 1, com o objetivo de ajudar os participantes a identificarem os elementos que desencadeiam insatisfação e problemas de adaptação acadêmica, bem como ajudá-los a criar estratégias para melhorar a adaptação e a satisfação acadêmica, adotando uma postura de agência pessoal, com base no referencial social cognitivo, na solução dos problemas acadêmicos e melhorando assim, os indicadores de adaptação acadêmica e adaptabilidade de carreira. 
Neste trabalho, entende-se o conceito de oficina como o atendimento em grupo com encontros delineados, com objetivos e procedimentos previamente definidos (Cupertino, 2008). Tendo em vista os objetivos previamente delineados, a oficina de adaptação acadêmica foi estruturada em oito encontros, sendo um por semana, com duração de 1 hora e 30 minutos cada. De modo geral, no $1^{\circ}$ encontro foram explicitados os objetivos da oficina, combinadas as regras de funcionamento e aplicados os instrumentos de medida utilizados para a avaliação pré-teste, as escalas de Ajustamento Acadêmico e Adaptabilidade de Carreira. Do $2^{\circ}$ ao $7^{\circ}$ encontro foram trabalhados diferentes temas associados à adaptação acadêmica e no último encontro foram reaplicados os instrumentos do pós-teste e encerrada a oficina.

As oficinas de adaptação acadêmica, analisadas neste estudo, foram realizadas por três semestres entre abril de 2016 e julho de 2017. Durante este período, avaliações do processo foram realizadas e adaptações foram feitas no modelo original de acordo com as sugestões dadas pelos participantes e coordenadores. Buscou-se analisar questões relacionadas ao tempo de execução das atividades propostas, bem como a adesão dos participantes na discussão das atividades e na avaliação do grupo a respeito da pertinência das atividades. Ao final de cada encontro, os participantes foram convidados a fazer uma avaliação das atividades realizadas e de suas possíveis contribuições.

Para a avaliação da intervenção foram comparados os resultados obtidos por meio dos instrumentos:
Questionário de Ajustamento Acadêmico e Escala de Adaptabilidade de Carreira, mencionados anteriormente, comparando-os nas situações de pré-teste e pós-teste. Para as análises quantitativas utilizou-se o programa estatístico Statistical Package for Social Sciences (SPSS) versão 23 no qual foram realizadas estatísticas descritivas e comparativas, tais como o cálculo da frequência, médias e ANOVA. Além disso, foi calculado o d de Cohen para conhecer o tamanho do efeito das diferenças, conforme as indicações feitas por Dancey e Reidy (2013).

\section{Resultados e Discussão}

\section{Delineamento e Implantação do Programa de Adaptação Acadêmica}

Como mencionado anteriormente, esta pesquisa foi desenvolvida em duas etapas. A primeira dedicada ao delineamento e à implantação de um programa de adaptação acadêmica no ensino superior e, a segunda dedicada a apresentar os resultados da intervenção. Em relação ao delineamento e implantação, o programa foi planejado no formato de oficina com oito encontros. E, o desenvolvimento se deu em três semestres. Ajustes e adaptações foram realizados após o encerramento de cada oficina de acordo com as necessidades sugeridas pelos participantes e coordenadores. A Tabela 1 mostra o modelo da oficina de adaptação acadêmica com a descrição dos objetivos de cada encontro e respectivas atividades.

Tabela 1

Primeiro Modelo da Oficina de Adaptação Acadêmica

\begin{tabular}{|c|c|c|}
\hline Encontros & Objetivos & Atividades \\
\hline Encontro 1 & $\begin{array}{l}\text { - Conhecer os participantes e suas expectativas } \\
\text { - Construir regras de funcionamento } \\
\text { - Aplicação do Pré-Teste }\end{array}$ & $\begin{array}{l}\text { - Atividade: Técnica do Barbante (ver Andrade, 1999) } \\
\text { - Pré-Teste (Questionário com as características } \\
\text { pessoais, socioeconômicas e acadêmicas; } \\
\text { Questionário de Ajustamento Acadêmico; e Escala } \\
\text { de Adaptabilidade de Carreira) }\end{array}$ \\
\hline Encontro 2 & $\begin{array}{l}\text { - Conhecer a história de vida dos participantes } \\
\text { - Identificar a relação dos indivíduos com a } \\
\text { cidade, com o curso e com a universidade }\end{array}$ & $\begin{array}{l}\text { - Atividade: Estrada da Formação } \\
\text { (ver www.dinamicaspassoapasso.com.br) }\end{array}$ \\
\hline Encontro 3 & $\begin{array}{l}\text { - Identificar e analisar os eventos rotineiros que } \\
\text { influenciam na dificuldade de adaptação }\end{array}$ & $\begin{array}{l}\text { - Atividade: Gosto e Faço (ver Lucchiari, 1993) } \\
\text { - Reflexões sobre vantagens e desvantagens da } \\
\text { cidade, do curso e da universidade } \\
\text { (elaborado pelas autoras) }\end{array}$ \\
\hline Encontro 4 & $\begin{array}{l}\text { - Analisar as dificuldades e perceber qual é o papel } \\
\text { delas na busca pela conquista dos objetivos }\end{array}$ & $\begin{array}{l}\text { - Atividade: Frases para Completar } \\
\text { (ver Lucchiari, 1993) }\end{array}$ \\
\hline
\end{tabular}


Tabela 1 (continuação)

Primeiro Modelo da Oficina de Adaptação Acadêmica

\begin{tabular}{|c|c|c|}
\hline Encontros & Objetivos & Atividades \\
\hline Encontro 5 & $\begin{array}{l}\text { - Orientar os participantes no que tange à } \\
\text { estratégia de organização do tempo, de modo } \\
\text { que percebam a responsabilidade da agência } \\
\text { pessoal nas atividades diárias }\end{array}$ & $\begin{array}{l}\text { - Atividade: Construção da agenda semanal e leitura } \\
\text { da Carta } 4 \text { do livro Gervásio ao seu Umbigo } \\
\text { (ver Rosário, Núnez, \& Gonzáles-Pienda, 2012) }\end{array}$ \\
\hline Encontro 6 & $\begin{array}{l}\text { - Refletir coletivamente sobre soluções } \\
\text { para os problemas vigentes } \\
\text { - Desenvolver estratégias para lidar } \\
\text { com o problema }\end{array}$ & $\begin{array}{l}\text { - Discutir se foi possível colocar em prática a agenda } \\
\text { - Atividade: Troca de um Segredo } \\
\text { (ver www.sato.adm.br) }\end{array}$ \\
\hline Encontro 7 & $\begin{array}{l}\text { - Evidenciar aos participantes que eles têm a } \\
\text { autonomia para controlar fatores internos que os } \\
\text { impedem de colocar seus objetivos em prática }\end{array}$ & $\begin{array}{l}\text { - Atividade: Leitura e discussão do texto sobre } \\
\text { a Lenda do Peixinho Vermelho (ver Monteiro, } \\
\text { Ventura \& Cruz, 2015) } \\
\text {-Atividade do Alvo (elaborado pelas autoras) }\end{array}$ \\
\hline Encontro 8 & - Avaliação Final do Processo & $\begin{array}{l}\text { - Atividade: Carta de despedida (ver Lucchiari, 1993) } \\
\text { - Avaliação Final do Programa; } \\
\text { - Pós-Teste (Questionário de Ajustamento } \\
\text { Académico, Escala de Adaptabilidade de Carreira }\end{array}$ \\
\hline
\end{tabular}

Ao longo de três semestres, foi verificada a necessidade de algumas adaptações no modelo inicial, de forma a torná-lo mais adequado à realidade e às demandas dos participantes. Nesse sentido e de forma processual, a proposta original passou por adaptações chegando assim, a última versão que se encontra descrita na Tabela 2.

Como estratégia para melhorar o processo de intervenção verificou-se a necessidade de pequenas modificações na ordem e nas atividades utilizadas. Mudanças foram feitas no $2^{\circ}$ encontro do primeiro modelo da oficina (ver Tabela 1), em que inicialmente era utilizada a atividade da "Estrada da Formação" (ver www.dinamicaspassoapasso. com.br). Para a conclusão desta técnica exigia-se dois encontros. Assim, como pode ser observado na Tabela 2, surgiu a necessidade adaptação da atividade, sendo esta substituída pela atividade "Linha da Vida" (elaborada pelas autoras) e, como tarefa para casa incluiu-se a atividade "Frases para Completar" (ver Lucchiari, 1993).

Tabela 2

Modelo Final da Oficina de Adaptação Acadêmica

\begin{tabular}{|c|c|c|}
\hline Encontros & Objetivos & Atividades \\
\hline Encontro 1 & $\begin{array}{l}\text { - Conhecer os participantes e suas expectativas } \\
\text { - Construir regras de funcionamento e } \\
\text { - Aplicação do Pré-Teste }\end{array}$ & $\begin{array}{l}\text {-Atividade: Dinâmica do Barbante (ver Andrade, 1999) } \\
\text { - Pré-Teste (Questionário com as características } \\
\text { pessoais, socioeconômicas e acadêmicas; } \\
\text { Questionário de Ajustamento Acadêmico; e Escala } \\
\text { de Adaptabilidade de Carreira) }\end{array}$ \\
\hline Encontro 2 & - Discussão e reflexão sobre a trajetória de vida & $\begin{array}{l}\text { - Atividade: Linha da vida (elaborada pelas autoras); } \\
\text {-Atividades para casa: Frases para completar } \\
\text { (ver Lucchiari, 1993) }\end{array}$ \\
\hline Encontro 3 & $\begin{array}{l}\text { - Compreender habilidades e perfis profissionais } \\
\text { dos participantes }\end{array}$ & $\begin{array}{l}\text { - Questionário de Interesses Vocacionais } \\
\text { (elaborado pelas autoras) }\end{array}$ \\
\hline
\end{tabular}


Tabela 2 (continuação)

Modelo Final da Oficina de Adaptação Acadêmica

\begin{tabular}{|c|c|c|}
\hline Encontros & Objetivos & Atividades \\
\hline Encontro 4 & $\begin{array}{l}\text { - Identificar e refletir coletivamente sobre } \\
\text { soluções para as problemáticas vigentes e } \\
\text { autoconhecimento }\end{array}$ & $\begin{array}{l}\text { - Atividade: Troca de um Segredo } \\
\text { (ver www.sato.adm.br) } \\
\text { - Atividade: Leitura e reflexão da Carta } 6 \text { do livro } \\
\text { Gervásio ao seu Umbigo (ver Rosário, Núnez, \& } \\
\text { Gonzáles-Pienda, 2012) }\end{array}$ \\
\hline Encontro 5 & $\begin{array}{l}\text { - Orientar os participantes no que tange à } \\
\text { estratégia de organização do tempo, de modo } \\
\text { que percebam a responsabilidade da agência } \\
\text { pessoal nas atividades diárias }\end{array}$ & $\begin{array}{l}\text { - Atividades: Gosto e Faço (ver Lucchiari, 1993) e, } \\
\text {-Construção da agenda semanal e leitura da carta } \\
4 \text { do livro Gervásio ao seu Umbigo (ver Rosário, } \\
\text { Núnez, \& Gonzáles-Pienda, 2012) }\end{array}$ \\
\hline Encontro 6 & $\begin{array}{l}\text { - Compreender as principais dificuldades } \\
\text { ou reorientação e desejos para o futuro } \\
\text { profissional }\end{array}$ & $\begin{array}{l}\text { - Discutir se foi possível colocar em prática a agenda. } \\
\text { - Atividade: Dinâmica árvore dos sonhos e muro das } \\
\text { lamentações (ver enactus s/d) }\end{array}$ \\
\hline Encontro 7 & $\begin{array}{l}\text { - Evidenciar aos participantes que eles têm a } \\
\text { autonomia para controlar fatores internos que os } \\
\text { impedem de colocar seus objetivos em prática }\end{array}$ & $\begin{array}{l}\text {-Atividade: Percepção do Ambiente (elaborado pelas } \\
\text { autoras) } \\
\text { - Leitura e discussão do texto sobre a Lenda do Peixinho } \\
\text { Vermelho (ver Monteiro, Ventura \& Cruz, 2015) } \\
\text { - Atividade do Alvo (elaborado pelas autoras) }\end{array}$ \\
\hline Encontro 8 & • Avaliação Final do Processo & $\begin{array}{l}\text { - Carta de despedida (ver Lucchiari, 1993) } \\
\text { - Avaliação Final do Programa } \\
\text { - Pós-Teste (Questionário de Ajustamento } \\
\text { Acadêmico, Escala de Adaptabilidade de Carreira) }\end{array}$ \\
\hline
\end{tabular}

Foi alterado também, como mostra a Tabela 2 , o $3^{\circ}$ encontro da oficina, em que surgiu a necessidade de acrescentar um novo tema, não abordado no primeiro modelo, para discutir os interesses e perfis profissionais dos participantes, já que estes apresentavam dúvidas em relação à escolha profissional realizada e apresentavam poucos conhecimentos sobre si mesmos e das possibilidades de exploração vocacional, com o objetivo de conhecer as ocupações e o mundo do trabalho.

Assim, a ordem da atividade "Frases para Completar" passou do $4^{\circ}$ encontro para o $2^{\circ}$ encontro (como tarefa para casa) devido à necessidade de um maior autoconhecimento dos participantes no início da oficina. Além disso, a tarefa do "Gosto e Faço" da vida acadêmica (ver Lucchiari, 1993) passou do $3^{\circ}$ para o $5^{\circ}$ encontro, pois esta técnica envolve um nível maior de autoconhecimento e exploração sobre habilidades e interesses profissionais, sendo melhor aproveitada pelos participantes do meio para o final da intervenção.

No $3^{\circ}$ encontro do modelo inicial da oficina (ver Tabela 1) pode-se observar que a atividade "Vantagens e
Desvantagens da cidade, do curso e da universidade", foi substituída pela atividade "Árvore dos Sonhos e Muro das Lamentações" (ver Enactus s/d.), como pode ser visto na Tabela 2, que passou a acontecer no $6^{\circ}$ encontro. Ambas as atividades tem o mesmo propósito de refletir sobre as vantagens e as desvantagens que permeiam a vida acadêmica, porém, a forma de abordar o tema ocorre de maneira diferente em cada proposição, sendo que a técnica "Árvore dos Sonhos e Muro das Lamentações" mais lúdica e interativa, não sendo apenas reflexiva, envolvendo a colaboração de todos os participantes.

Ainda, a atividade "Troca de um Segredo" (ver www. sato.adm.br) passou do $6^{\circ}$ encontro para o $4^{\circ}$, pois seu objetivo é auxiliar na resolução de um problema, o que fez esta temática ser adiantada para o começo da oficina com o intuito de que todos os participantes se ajudassem na resolução dos problemas e aprendessem recursos para solucionar outros problemas, assim haveria mais tempo para a elaboração do conteúdo no decorrer dos demais encontros da oficina. Além disso, incluiu-se no $4^{\circ}$ encontro a "Leitura e reflexão da Carta 6 do livro "Gervásio ao seu Umbigo" (ver Rosário, Núnez, \& Gonzáles-Pienda, 
2012), que trata do tema autorregulação da aprendizagem e do sucesso acadêmico.

Por fim, no modelo final da oficina (Tabela 2) observa-se no $7^{\circ}$ encontro a inclusão de uma nova atividade de aquecimento, elaborada pelas autoras, sobre "Percepção do Ambiente", que teve por objetivo convidar os participantes a observarem e descreverem o ambiente que estavam. Esta atividade foi planejada para contextualizar e auxiliar em discussões mais aprofundadas sobre o texto base do encontro a "Lenda do Peixinho Vermelho" (ver Monteiro, Ventura \& Cruz, 2015). Nessa atividade os participantes foram estimulados a pensar sobre o comportamento exploratório, por meio da história de um peixe que desbrava o mar com esforço e, o que ele proporciona de bom e perigoso, em analogia a exploração de carreira, oportunidades, ter a visão mais ampla sobre o futuro e seus esforços pessoais.

Frente aos resultados obtidos nesta etapa constatou-se que o formato de oficina com oito encontros foi adequado para atender aos objetivos propostos no programa. A avaliação dos participantes e os registros das coordenadoras das oficinas possibilitaram analisar o processo de intervenção durante e após sua execução contribuindo para a melhoria gradual do programa. Nessa direção, Bardagi e Albanaes (2015) ressaltam a importância da avaliação contínua dos processos de Orientação Profissional e Carreira, no intuito de estabelecer possíveis adaptações no modelo da intervenção de modo a garantir a melhoria continua do processo.

\section{Avaliação dos Resultados da Intervenção}

Finalizada a etapa do delineamento e da implantação do programa, foi realizada a avaliação dos resultados da intervenção. Para atingir este objetivo comparou-se os resultados das respostas dos participantes aos dois instrumentos de medida pré-teste e pós-teste: (a) Questionário de Ajustamento Acadêmico: em suas dimensões relacionadas à disposição afetiva, a autoeficácia acadêmica, a percepção de alcance e progresso em objetivos acadêmicos, de recursos ou barreiras, a satisfação com a vida acadêmica, o estresse percebido com a vida acadêmica, a percepção de adaptação geral à universidade e a satisfação com a vida em geral; e (b) Escala de Adaptabilidade de Carreira: em suas dimensões relacionadas à preocupação com a carreira, percepção de controle, curiosidade e confiança. A Tabela 3 mostra os resultados obtidos por meio da Escala de Ajustamento Acadêmico.

Tabela 3

Resultados da comparação pré-teste e pós teste na Escala de Ajustamento Acadêmico

\begin{tabular}{|c|c|c|c|c|c|c|}
\hline \multicolumn{7}{|c|}{ Escala de Ajustamento Acadêmico } \\
\hline & \multicolumn{2}{|c|}{ Pré-teste } & \multicolumn{2}{|c|}{ Pós-teste } & \multirow{2}{*}{$\mathrm{T}$} & \multirow{2}{*}{$\mathrm{P}$} \\
\hline & Média & DP & Média & DP & & \\
\hline Autoeficácia acadêmica & 5,85 & 1,47 & 6,61 & 1,41 & $-2,566$ & $0,018^{*}$ \\
\hline Autoeficácia para lidar com barreiras & 5,77 & 1,17 & 5,85 & 1,33 & $-0,227$ & 0,823 \\
\hline Percepção de progresso e alcance dos objetivos acadêmicos & 3,12 & 0,61 & 3,22 & 0,57 & $-0,687$ & 0,500 \\
\hline Percepção de apoio social & 3,54 & 0,57 & 3,66 & 0,43 & $-1,185$ & 0,249 \\
\hline Satisfação com a vida acadêmica & 3,14 & 0,57 & 3,14 & 0,42 & $-0,058$ & 0,954 \\
\hline Percepção de adaptação geral a universidade & 2,68 & 0,77 & 2,81 & 0,66 & $-1,142$ & 0,266 \\
\hline Estresse percebido com a vida acadêmica & 3,21 & 0,45 & 3,18 & 0,39 & 0,287 & 0,777 \\
\hline Disposição afetiva & 2,81 & 0,54 & 2,97 & 0,45 & $-1,442$ & 0,164 \\
\hline Satisfação geral com a vida & 3,53 & 0,73 & 3,98 & 0,95 & $-2,359$ & $0,028 *$ \\
\hline
\end{tabular}

* Nota: grau de significância adotado como $p<0,05$

Como pode ser observado na Tabela 3, os participantes da Oficina de Adaptação Acadêmica conseguiram melhorar seus escores na maioria das dimensões da Escala de Ajustamento Acadêmico, em duas dimensões: "Autoeficácia Acadêmica" e "Satisfação Geral com a Vida", os ganhos foram significativos. Foram observados acréscimos significativos nas crenças de "Autoeficácia Acadêmica", após a intervenção $[t(21)=-2,566 ; p=$
0,018; I.C. a 95\%;]. Adicionalmente, o indicador d de Cohen mostrou que o efeito da diferença entre as médias antes e após a intervenção foi de intensidade média $(d=-0,53)$. Estes resultados mostram que os participantes se sentiam, após a intervenção, mais confiantes em manter-se envolvidos nos seus cursos ao longo dos próximos semestres. Os registros no diário de campo mostram que os participantes relataram empenho ao longo do semestre, 
"dando ao máximo de si", para conseguirem completar as disciplinas nas quais estavam matriculados, obtendo classificação final igual ou superior ao exigido na universidade. Estes resultados são importantes no contexto da adaptação acadêmica, foco do programa de intervenção e objeto deste estudo.

Sabe-se que a "Autoeficácia Acadêmica" refere-se à crença que o estudante tem na sua capacidade para organizar e pôr em prática ações referentes a todas as atividades e exigências acadêmicas, relacionadas às habilidades cognitivas na construção de novos conhecimentos. Assim, os avanços obtidos pelos participantes ao final da intervenção vão de encontro ao que destaca a literatura, uma vez que a autoeficácia tem sido considerada atualmente como uma das grandes dimensões psicológicas responsáveis pelo desempenho acadêmico e pela persistência nas atividades a serem cumpridas ao longo da graduação, influenciando na adaptação acadêmica dos universitários (Joly, Serpa, Borges \& Martins, 2016). Assim, a autoeficácia elevada relaciona-se a um alto nível de motivação, podendo ter como consequência maior esforço, persistência perante as dificuldades e obstáculos, influenciando fortemente na adaptação acadêmica desses universitários, pois um aluno sente-se motivado a envolver-se no processo de aprendizagem se acreditar que seus conhecimentos, aptidões e capacidade vão permitir-lhe aprender novos conteúdos e aperfeiçoar novas competências (Meneses \&Silva, 2009).

Para que melhores níveis de "Autoeficácia Acadêmica" fossem atingidos, durante o processo de intervenção, foco deste estudo, foram realizadas atividades direcionadas a este fim, principalmente nos encontros que exploravam estratégias para melhorar a administração do tempo e estimular a agência pessoal. Esses encontros tinham como objetivo trabalhar a autorregulação - para ativá-la -, e procrastinação - para compreendê-la e diminuí-la - bem como desenvolver métodos de estudos. E, assim tronar-se agente de sua própria vida.
Nota-se também, por meio dos resultados apresentados na Tabela 3, que houve aumento significativo dos indicadores de "Satisfação Geral com a Vida", após a intervenção $[t(21)=-2,359 ; p=0,028$; IC. a 95\%]. Nessa dimensão, o efeito da diferença entre as médias antes e após a intervenção foi de intensidade média $(d$ $=-0,54)$. Ou seja, os resultados sugerem que os participantes tornaram-se mais satisfeitos com a vida ao final da intervenção.

A "Satisfação Geral com a Vida" diz respeito à avaliação cognitiva que o estudante faz sobre sua qualidade de vida geral. Essa avaliação geralmente é realizada a partir da comparação entre os aspectos de vida atual do acadêmico e um padrão que este determina para si como ideal (Bardagi \& Hutz, 2010). A percepção de satisfação com a vida em geral contribui para um maior bem-estar psíquico e social do estudante, sendo também relevante para sentimentos de autorrealização, refletindo assim na importância da percepção positiva de si, maior nível de independência e capacidade para estabelecer relações de confiança e adaptação às exigências do meio acadêmico.

Os registros no diário de campo possibilitaram informar que durante a oficina, em vários encontros foram feitos questionamentos relacionados ao ideal de vida, satisfação e conquistas de vida, atividades de autoconhecimento, vantagens e desvantagens na vida acadêmica e geral, elaboração de metas para objetivos futuros e desejos dos mais variados âmbitos da vida. Assim, essas questões foram trabalhadas com os participantes, pois foram temáticas emergentes e estavam em consonância com os objetivos do programa.

Em relação à "Adaptabilidade de Carreira", nota-se por meio dos resultados apresentados na Tabela 4 que os participantes conseguiram melhorar seus escores em todas as dimensões do constructo, sendo que em três dimensões as diferenças entre as médias, antes e após a intervenção, foram significativas.

Tabela 4

Resultados da comparação pré-teste e pós-teste Escala de Adaptabilidade de Carreira

\begin{tabular}{|c|c|c|c|c|c|c|}
\hline \multicolumn{7}{|c|}{ Escala de Adaptabilidade de Carreira } \\
\hline & \multicolumn{2}{|c|}{ Pré-teste } & \multicolumn{2}{|c|}{ Pós-teste } & \multirow{2}{*}{$\mathrm{T}$} & \multirow{2}{*}{$\mathrm{P}$} \\
\hline & Média & DP & Média & DP & & \\
\hline Preocupação com a carreira & 3,06 & 0,85 & 3,81 & 0,59 & $-4,506$ & $0,000^{*}$ \\
\hline Controle & 3,22 & 0,58 & 3,56 & 0,64 & $-2,241$ & $0,036^{*}$ \\
\hline Curiosidade & 2,96 & 0,69 & 3,46 & 0,57 & $-2,677$ & $0,014 *$ \\
\hline Confiança & 3,09 & 0,51 & 3,42 & 0,70 & $-1,912$ & 0,070 \\
\hline
\end{tabular}

* Nota: grau de significância adotado como $p<0,05$ 
Acréscimos significativos foram observados nos níveis da dimensão "Preocupação com a Carreira", após a intervenção $[t(21)=-4,506 ; p=0,000$; I.C. a 95\%]. Nesta dimensão, o efeito da diferença entre as médias antes e após a intervenção foi alto $(d=-1,04)$. Isso indica que após a intervenção, os estudantes estavam mais reflexivos em relação ao futuro, percebendo que as conquistas do futuro dependem das escolhas do presente, atentando-se às escolhas educacionais e profissionais que fazem e planejando metas para atingir os objetivos que desejam.

A "Preocupação com a Carreira", uma das quatro dimensões principais da "Adaptabilidade de Carreira", refere-se à preocupação dos participantes em relação ao seu próprio futuro como trabalhador (Savickas, 2005). Ou seja, leva o indivíduo a possuir um maior senso de orientação quanto ao futuro vocacional, em que este tenha atitudes de planejamento, antecipação e preparação em relação a sua carreira e profissão (Audibert \& Teixeira, 2015). Além disso, essa maior preocupação auxilia também na percepção de que o passado, o presente e o futuro estão relacionados e que atividades de escolhas desempenhadas atualmente influenciam futuros projetos profissionais, ou seja, a preocupação central com a carreira e o planejamento de carreira induz a capacidade para ligar as atividades presentes ao futuro desejado (Koen, Klehe \& Vianen, 2012). Audibert e Teixeira (2015) citam que a falta de "preocupação com a carreira" acarreta em indiferença, tendo como consequência, a ausência de planos e o pessimismo em relação ao futuro, o que interfere nas demais dimensões da adaptabilidade.

Adicionalmente, após a intervenção foram observadas diferenças significativas e de intensidade moderada $(d=-0,51)$ em relação à dimensão "Controle" $[t(21)=-2,241 ; p=0,036$ I.C. a 95\%]. Estes resultados informam que após a intervenção os participantes mostravam-se mais entusiasmados e otimistas, tomando decisões por conta própria, assumindo responsabilidade pelos atos, acreditando assim na capacidade de dirigirem as próprias vidas, fazendo o que consideram certo para eles.

A dimensão "Controle" baseia-se na capacidade do estudante de não apenas utilizar estratégias de autorregulação para ajustar-se às demandas dos diferentes contextos, em especial acadêmico e profissional, mas também exercer algum tipo de influência e controle sobre suas vidas e decisões de carreira (Duarte et al., 2009). Os ganhos na dimensão "Controle", após a intervenção sinalizam que os participantes do programa adquiriram habilidades para controlar as tentativas de se preparar para o futuro profissional e de carreira, assumindo responsabilidades em seus comportamentos e ambientes por meio de autodisciplina, esforço e persistência (Ambiel, 2014). Ou seja, os participantes, após a intervenção, sentiam-se mais responsáveis por construir a sua carreira, assumindo uma postura ativa no sentido de fazer escolhas e determinar o futuro profissional. Esta postura de maior controle é útil no contexto da adaptação acadêmica, pois leva os indivíduos ao enfrentamento das questões vocacionais de forma assertiva ao invés de conduzir-se à procrastinação (Audibert \& Teixeira, 2015). Este resultado vai ao encontro do que ressaltam Ambiel, Santos e Dalbosco (2016) sobre a competência dos estudantes de se responsabilizarem pelas próprias decisões de carreira, o que diminui o uso dos motivos relacionados à dependência de outros para se manter no curso, assumindo assim sua real decisão.

Ainda, tendo em vista os dados apresentados na Tabela 4, observou-se diferença significativa e de intensidade moderada $(d=-0,79)$ em relação à dimensão "Curiosidade", após a intervenção $[t(21)=-2,667$; $p=0,014$ I.C. a 95\%]. Esse resultado está em consonância com os objetivos originais da intervenção, que buscou ajudar os participantes a explorar o ambiente acadêmico, procurando disponibilizar oportunidades de crescimento pessoal, de exploração de opções antes de fazerem outras escolhas, sendo curiosos em relação às novas oportunidades e investigando profundamente as dúvidas.

A dimensão "Curiosidade" relaciona-se com a exploração de si mesmo e das possibilidades futuras, a capacidade de se colocar em várias situações e papéis, ou seja, comportamentos de exploração ativa e estar adaptado ao dinamismo do ambiente profissional no qual o estudante estará inserido brevemente. A "Curiosidade" na construção da carreira ajuda as pessoas a formarem uma imagem realista de si mesmo e suas opções de carreira. Este conhecimento facilita escolhas subsequentes que combinam o mesmo com situações ocupacionais (Koen et al., 2012).

No escopo do programa de adaptação acadêmica, algumas atividades propostas ao longo da intervenção podem ter sido úteis para o aumento registrado da "Adaptabilidade de Carreira", em especial as tarefas e discussões ligadas à construção da Linha da Vida, que interligou as dimensões de passado, presente e futuro, possibilitando o estabelecimento de metas e planos de ação para realizá-las no futuro profissional. Em relação à exploração de si e do ambiente foram trabalhados os desejos e as inseguranças dos participantes em relação a sua adaptação acadêmica, bem como a importância do comportamento exploratório fortalecendo as dimensões da Preocupação, Controle e Curiosidade. 


\section{Conclusões e Considerações Finais}

Esta pesquisa teve como objetivo apresentar o processo de delineamento e implantação de um programa de adaptação acadêmica no ensino superior e os resultados dele decorrentes, em termos de processo e resultados. A avaliação processual permitiu disponibilizar um modelo de serviço que atende às demandas das instituições de ensino superior no que diz respeito à oferta de serviços de orientação acadêmica destinados a facilitar a adaptação acadêmica e, assim, promover a saúde de futuros profissionais de nível superior.

Além disso, os resultados obtidos após a realização do programa foram considerados satisfatórios. Os achados desta investigação reforçam as evidências obtidas em outros estudos que demonstraram satisfação dos usuários com programas de orientação acadêmica quanto ao desenvolvimento de habilidades, ao estabelecimento de objetivos realistas, ao reconhecimento de seus valores, ao desenvolvimento de habilidades para trabalhar em equipe, à organização um projeto de vida e a tomar a decisão profissional, como apontado por Arruda e Melo-Silva (2010) em um estudo com egressos de um serviço de orientação profissional com jovens em situação de vestibular.

Adicionalmente, a avaliação dos resultados do programa sinalizou que houve aumentos significativos nos resultados relativos à "Autoeficácia Acadêmica", "Satisfação geral com a Vida", "Preocupação com a Carreira", "Controle" e "Curiosidade", após a intervenção. Dessa forma, é possível dizer que os participantes conseguiram desenvolver importantes recursos psicossociais que serão úteis para o enfrentamento de situações que se apresentam como desafios no processo de adaptação acadêmica, melhorando a capacidade de resolução de problemas típicos desta fase da vida e carreira.

Além das contribuições teóricas e práticas a presente investigação sinaliza importantes contribuições metodológicas para a prática e a pesquisa no campo Orientação Profissional e de Carreira, uma vez que para o alcance dos objetivos foram utilizados múltiplos métodos, como a pesquisa-ação para a coleta dos dados e o método quase-experimental para a análise. Apesar das contribuições observadas, algumas limitações podem ser apontadas. A primeira delas foi a impossibilidade de realizar análises comparativas entre grupos controle e experimental, etapa importante para reforçar as evidências de eficácia do programa. Contudo, no decorrer da pesquisa-ação houve dificuldade para ampliar o tamanho da amostra contemplando concomitantemente a formação dos dois grupos. Adicionalmente, não foi possível replicar a intervenção em outros contextos universitários. Nesse sentido, sugere-se a continuidade das investigações com o modelo de oficina proposto, buscando replicar o estudo em outros contextos e que se faça uso de grupos controle e experimental como estratégia para aumentar as evidências de validade do programa apresentado. Por fim, espera-se que esta pesquisa sirva de referência para outras investigações que busquem delinear e avaliar intervenções direcionadas à facilitação da adaptação acadêmica no ensino superior e à promoção da saúde.

\section{Referências}

Almeida, L. S. (2007). Transição, adaptação acadêmica e êxito escolar no ensino superior. Revista Galelo-Portuguesa de Psicologia e Educación, 15(2), 203-215.

Almeida, L. S., Araújo, A. M., \& Martins, C. (2016). Transição e adaptação dos alunos do $1^{\circ}$ ano: Variáveis intervenientes e medidas de atuação. $1^{\circ}$ Seminário "Ser Estudante no Ensino Superior: O caso dos estudantes do $1^{\circ}$ ano", 146-164.

Almeida, F. H.; Melo-Silva, L. L. (2006). Avaliação de um serviço de Orientação Profissional: A perspectiva de exusuários. Revista Brasileira de Orientação Profissional 7(2), p. 81-100.

Alonso, W. C., \& Melo-Silva, L. L. (2013). Avaliação de uma intervenção em orientação profissional na perspectiva de ex-estagiários. Psicologia: Ciência e Profissão, 33(1), 84-99. doi: 10.1590/S1414-98932013000100008

Ambiel, R. A. M. (2014). Adaptabilidade de carreira: Uma abordagem histórica de conceitos, modelos e teorias. Revista Brasileira de Orientação Profissional, 15(1), 15-24.

Ambiel, R. A. M., Santos, A. A. A., \& Dalbosco, S. N. P. (2016). Motivos para evasão, vivências acadêmicas e adaptabilidade de carreira em universitários. Psico (Porto Alegre), 47(4), 288-297. doi: 10.15448/1980-8623.2016.4.23872

Arruda, M. N. F., \& Melo-Silva, L. L. (2010). Avaliação da intervenção de carreira: A perspectiva dos ex-clientes. Psico-USF, 15(2), 225-234. doi: 10.1590/s1413-82712010000200010

Audibert, A., \& Teixeira, M. A. P. (2015). Escala de adaptabilidade de carreira: Evidências de validade em universitários brasileiros. Revista Brasileira de Orientação Profissional, 16(1), 83-93.

Bardagi, M. P., \& Albanaes, P. (2015). Avaliação de intervenções vocacionais no Brasil: Uma revisão da literatura. Revista Brasileira de Orientação Profissional, 16(2), 123-135. 
Bardagi, M. P., \& Hutz, S. C. (2010). Satisfação de vida, comprometimento com a carreira e exploração vocacional em estudantes universitários. Arquivos Brasileiros de Psicologia, 62(1), 159-170.

Berhein, C. T., \& Chauí, M. (2008). Desafios da universidade na sociedade do conhecimento cinco anos depois da conferência mundial sobre educação superior. Brasília: UNESCO.

Bogdan, R., \& Biklen, S. (1994). Investigação qualitativa em educação. Porto: Porto Editora.

Byrd, D. R., \& McKinney, K. J. (2012). Individual, interpersonal, and institutional level factors associated with the mental health of college students. Journal of American College Health, 60(3), 185-193. doi: 10.1080/07448481.2011.584334

Cozby, P. C. (2009). Métodos de Pesquisa em Ciências do Comportamento. São Paulo: Atlas.

Cupertino, C. M. B. (2008). Espaços de criação em psicologia: Oficinas na prática. São Paulo: Anablume.

Dancey, C. P., \& Reidy, J. (2013). Estatísticas em matemática para psicologia: usando SPSS para Windows [Statistics with outmathematics to psychology: using SPSS for Windows]. Porto Alegre, RS: Artmed.

Dinâmica de grupo - Troca de um Segredo (s/d.). Recuperado em 27 de janeiro de 2018, em http://www.sato.adm.br/rh/ dinamica_de_grupo_troca_de_um_segredo.htm

Dinâmica para desenvolvimento pessoal e autoconhecimento - A Estrada (2011). Recuperado em 27 de janeiro de 2018, em http://www.dinamicaspassoapasso.com.br

Duarte, M. E., Lassance, M. C., Savickas, M. L., Nota, L., Rossier, J., D., J., Guichard, J, Esbroeck, R. V., \& Vianen A. E. M. (2009). Construção da vida: um novo paradigma para entender a carreira no século XXI. Revista Interamericana de Psicologia, 44(2), 392-406. doi: 10.1016/j.jvb.2009.04.004

Enactus. (s/d.). Diagnóstico Participativo. Oficina árvore dos sonhos e muro das lamentações. Recuperado em 27 de janeiro de 2018, em /wp-content/uploads/sites/2/2017/01/2.-Diagnóstico-Participativo.pdf

Fagundes, C.V., Luce, M. B., \& Rodriguez Espinar, S. (2014). O desempenho acadêmico como indicador de qualidade da transição ensino médio-educação superior. Ensaio: Avaliação e Políticas Públicas em Educação, 22(84), 635-669. doi: $10.1590 / \mathrm{s} 0104-40362014000300004$

Freitas, O. A., \& Oliveira, M. C. (2017). Trajetória, projetos e expectativas de sucesso na carreira: estudo com universitários concluintes que não pretendem atuar na área de formação. Revista Brasileira de Ensino Superior, 3(2), 58-78. doi: 10.18256/2447-3944.2017.v3i2.1555

Guerreiro-Casanova, D. C., \& Polydoro, S. A. J. (2011). Integração ao ensino superior: Relações ao longo do primeiro ano de graduação. Psicologia: Ensino \& Formação, 1(2): 85-96.

Instituto Nacional de Estudos e Pesquisas Educacionais Anísio Teixeira (INEP). (2016). Ministério da Educação. Censo da educação superior: 2015 - Sinopses estatísticas da educação superior - graduação. Recuperado em 22 de novembro de 2016, http://download.inep.gov.br/educacao_superior/censo_superior/documentos/2015/Notas_ Estatisticas_Censo_Superior_2015.pdf

Joly, M. C. R.A., Serpa,A. L. O., Borges, L., Martins, R. M. M. (2016). Autoeficácia acadêmica e autorregulação da aprendizagem: Rede de relacionamento em bases online. Avaliação Psicológica, 15(1), 73-82. doi: 10.15689/ap.2016.1501.08

Koen, J., Klehe, U., \& Viannen, A. E. M. (2012). Training career adaptability to facilitate a successful school-to-work transition. Journal of Vocational Behavior, 81, 395-408. doi: 10.1016/j.jvb.2012.10.003

Lent, R.W. (2004). Toward a unifying theoretical and practical perspective on well-being and psychosocial adjustment. Journal of Counseling Psychology, 51, 482-509. doi: 10.1037/0022-0167.51.4.482

Lent, R.W., Singley, D., Sheu, H., Gainor, K., Brenner, B.R., Treistman, D., \& Ades. L. (2005). Social cognitive predictors of domain and life satisfaction: Exploring the theoretical precursors of subjective well-being. Journal of Counseling Psychology, 52, 429-442. doi: 10.1037/0022-0167.52.3.429

Lent, T. W., Taveira, M. C., Cristiane, V., Sheu, H. B., \& Pinto, J. C. (2018). Test of the social cognitive model well-being in Portuguese and Brazilian college students. Journal of Vocational Behavior, 109, 78-86. doi:10.1016/j.jvb.2018.09.009

Lent, R.W., Taveira, M. C., Sheu, H., \& Singley, D. (2009). Social cognitive predictors of academic adjustment and life satisfaction in Portuguese college students: A longitudinal analysis. Journal of Vocational Behavior, 74, 190-198. doi: 10.1016/j.jvb.2008.12.006

Lucchiari, D. H. P. S. (Ed.). (1993). Pensando e vivendo a orientação profissional. São Paulo: Summus.

Melo-Silva, L. L., \& Jacquemin, A. (2001). Intervenção em Orientação Vocacional / profissional: Avaliando resultados e processos. São Paulo: Vetor Editora.

Melo-Silva, L. L. (2011). Intervenção e avaliação em orientação profissional. Em M. A. Ribeiro \& L. L. Melo-Silva (Orgs.). Compêndio de Orientação Profissional e de Carreira (vol. 2, pp. 155-192). São Paulo: Vetor. 
Meneses, R. F., \& Silva, I. (2009). Auto-eficácia de estudantes do $1^{\circ}$ ano de cursos superiores de saúde pós-Bolonha. Revista da Faculdade de Ciências da Saúde, 474-484.

Monteiro, C. D. B., Ventura, E. C., \& Cruz, P. N. (2015). Cultura e mudança organizacional. Recuperado em 27 de janeiro de 2018, de http://www.rhportal.com.br/artigos-rh/cultura-e-mudana-organizacional/

Nota, L., Soresi, S., Ferrari, L., \& Ginevra, M. C. (2014).Vocational designing and career counseling in Europe: Challenges and new horizons. European Psychologist, 19(4), 248-259. doi: 10.1027/1016-9040/a000189

OECD (2015). Education at a Glance 2015: OECD Indicators. OECD Publishing, Paris.

Oliveira, M. C., Detomini, V. C., \& Melo-Silva, L. L. (2013). Sucesso na transição universidade-trabalho: Expectativas de universitários formandos. Psicologia em Revista, 19(3), 497-518. doi: 10.5752/p.1678-9563.2013v19n3p497

Oliveira, C. T., Santos, A. S., \& Dias, A. C. G. (2016). Expectativas de universitários sobre a universidade: Sugestões para facilitar a adaptação acadêmica. Revista Brasileira de Orientação Profissional, 17(1), 43-53.

Oliveira, R. E. C., \& Morais. A. (2015). Vivências acadêmicas e adaptação de estudantes de uma universidade pública federal do Estado do Paraná. Revista Educação Pública. 57(24), 547-568.

Rosário, P., Núñez, J. C., \& González-Pienda, J. (2012). Cartas do Gervásio ao seu umbigo: Comprometer-se com o estudar na educação superior (S. A. J. Polydoro, Trad). São Paulo: Editora Almedina.

Savickas, M. L. (2005). The theory and practice of career construction. In: S. D. Brown \& R. W. Lent (Orgs). Career development and counseling: putting the oryan dresearch to work. Nova Jersey: John Wiley\& Sons, Inc.

Soares, A. B., \& Prette, Z. A. P. D. (2015). Habilidades sociais e adaptação à universidade: Convergências e divergências dos construtos. Análise Psicológica, 33(2), 139-151.

Teixeira, M. A. P., \& Gomes, W. B. (2004). Estou me formando...e agora?: Reflexões e perspectivas de jovens formandos universitários. Revista Brasileira de Orientação Profissional, 5(1), 47-62.

World Health Organization (WHO). (2017). Depression and other common mental disorders: Global health estimates.

Recebido 07/02/2018

Reformulado 01/09/2018

Aceite Final 07/10/2018

Sobre as autoras

Marcela de Moura Franco Barbosa é psicóloga, graduada em Psicologia pela Universidade Federal do Triângulo Mineiro (UFTM), participante do Grupo de Pesquisa em Educação e Construção da Carreira (GPECC) e do projeto de extensão intitulado Oriente-se: serviço de orientação acadêmica e de carreira, relacionada à adaptação acadêmica.

Marina Cardoso de Oliveira é psicóloga, doutora em Psicologia. Professora do curso de Psicologia da Universidade Federal do Triângulo Mineiro (UFTM). Coordenadora do Laboratório e do Grupo de Pesquisa em Educação e Construção da Carreira (LaPECC/GPECC).

Lucy Leal Melo-Silva é psicóloga, docente aposentada do Departamento de Psicologia da Faculdade de Filosofia, Ciências e Letras de Ribeirão Preto (FFCLRP-USP). Orienta pesquisas na Pós-Graduação. Membro da ABOP e da IAEVG. Coeditora da Revista Brasileira de Orientação Profissional. Publica no domínio da Orientação Profissional e de Carreira.

Maria do Céu Taveira é doutorada em Psicologia da Educação. Docente e investigadora da Escola de Psicologia da Universidade do Minho, Portugal. Coordena o grupo de investigação em Desenvolvimento de Carreira e Aconselhamento no Centro de Investigação em Psicologia (CIPsi). É autora e coautora de diversas publicações nacionais e internacionais nestes domínios. 